PROCEEDINGS OF THE

AMERICAN MATHEMATICAL SOCIETY

Volume 127, Number 10, Pages 2911-2915

S 0002-9939(99)04888-1

Article electronically published on April 23, 1999

\title{
A BESSEL FUNCTION MULTIPLIER
}

\author{
DANIEL OBERLIN AND HART F. SMITH \\ (Communicated by Christopher D. Sogge)
}

\begin{abstract}
We obtain nearly sharp estimates for the $L^{p}\left(\mathbb{R}^{2}\right)$ norms of certain convolution operators.
\end{abstract}

For $n \geq 1$ let $\lambda_{n}$ be the measure on $\mathbb{R}^{2}$ obtained by multiplying normalized arclength measure on $\{|x|=1\}$ by the oscillating factor $e^{i n \arg (x)}$. For $1 \leq p \leq \infty$, let $C(p, n)$ denote the norm of the operator $T_{n} f \doteq \lambda_{n} * f$ on $L^{p}\left(\mathbb{R}^{2}\right)$. The purpose of this note is to estimate the rate of decay of $C(p, n)$ as $n \rightarrow \infty$. By duality, it is enough to consider $p \geq 2$. Examples below will show that

$$
C(p, n) \geq C(p) n^{-\frac{1}{6}-\frac{1}{3 p}} \quad \text { if } \quad 2 \leq p \leq 4,
$$

and

$$
C(p, n) \geq C(p) n^{-\frac{1}{p}} \quad \text { if } \quad 4 \leq p \leq \infty .
$$

On the other hand, we will observe that

$$
\begin{aligned}
C(2, n) & \leq C n^{-\frac{1}{3}} \\
C(\infty, n) & \leq C
\end{aligned}
$$

and then prove the following result.

Theorem. There is a positive number a such that

$$
C(4, n) \leq C n^{-\frac{1}{4}}(\log (n))^{a} .
$$

Interpolating (3) and (4) gives upper bounds for $C(p, n)$ which differ only by a power of $\log (n)$ from the lower bounds of (1) and (2), thus providing nearly sharp estimates for $C(p, n)$.

The above question naturally arises when considering the $L^{p}\left(\mathbb{R}^{3}\right)$ mapping properties of the operator $T$ given by convolution with respect to a compact piece of arclength measure on the helix

$$
t \rightarrow(\cos t, \sin t, t) .
$$

$T$ is an example of a folding Fourier integral operator in dimension 3, whose singular set is of dimension 1 . The sharp $L^{p} \rightarrow L^{2}$ mapping properties of $T$ were established by the first author in $[\mathrm{O}]$. The operator $T_{n}$ arises when considering the

Received by the editors December 15, 1997.

1991 Mathematics Subject Classification. Primary 42B15, 42B20.

Key words and phrases. Fourier transform, convolution operator, oscillatory integral, Bessel function.

Both authors are partially supported by the NSF.

(C)1999 American Mathematical Society 
$L^{p}$ smoothing properties of $T$; that is, for which values of $\alpha_{p}$ is $|D|^{\alpha_{p}} T$ bounded on $L^{p}\left(\mathbb{R}^{3}\right)$. Since

$$
T\left(e^{-i n x_{3}} f\left(x_{1}, x_{2}\right)\right)=e^{-i n x_{3}}\left(T_{n} f\right)\left(x_{1}, x_{2}\right),
$$

the exponents in (1) and (2) give upper bounds on $\alpha_{p}$. In particular, the smoothing exponent for $T$ is less than that of averaging in $\mathbb{R}^{2}$ over the cubic $t \rightarrow\left(t, t^{3}\right)$, where the corresponding value of $\alpha_{p}$ is

$$
\alpha_{p}= \begin{cases}\frac{1}{3} \quad \text { if } \quad 2 \leq p<3, \\ \frac{1}{p} \quad \text { if } \quad 3<p<\infty .\end{cases}
$$

See, for example, [SW] or [SS]. The authors would like to thank Chris Sogge for discussions which led to consideration of this question.

To see (2), apply the operator $T_{n} f \doteq \lambda_{n} * f$ to $f(x)=e^{-i n \arg (x)} \chi_{A}(x)$ where $A$ is the annulus $\{1 \leq|x| \leq 1+1 / n\}$. One observes that there is a constant $C$ such that $\left|T_{n} f(x)\right| \geq C$ if $|x| \leq C / n$ and (2) follows (for all $p$, but (1) is better for $p \leq 4)$.

The example for (1) is a little more complicated: for fixed $n$, and $1 \leq j \leq n^{1 / 3}$, let $\theta_{j}=j n^{-1 / 3}, \omega_{j}=\left(\cos \left(\theta_{j}\right), \sin \left(\theta_{j}\right)\right)$, and $\omega_{j}^{\prime}=\left(-\sin \left(\theta_{j}\right), \cos \left(\theta_{j}\right)\right)$. Let $B_{j}$ be the disk $\left\{\left|x-\omega_{j}\right| \leq \varepsilon n^{-\frac{1}{3}}\right\}$ where $\varepsilon$ is a positive number independent of $n$ and small enough to insure that, for any $n$, the disks $B_{j}$ are pairwise disjoint. Let

$$
f_{j}(x)=e^{i n\left(x \cdot \omega_{j}^{\prime}\right)} \chi_{B_{j}}(x)
$$

One can check that

$$
\left|T_{n} f_{j}(x)\right| \geq c n^{-\frac{1}{3}} \quad \text { if } \quad|x| \leq c n^{-\frac{1}{3}}
$$

for some small positive $c$ independent of $n$ and $j$. Let $r_{j}$ be the $j$ th Rademacher function on $[0,1]$ and put

$$
f(t, x)=\sum_{j=1}^{n^{\frac{1}{3}}} r_{j}(t) f_{j}(x) .
$$

Then

$$
\|f(t, \cdot)\|_{p} \leq C n^{-\frac{1}{3 p}}
$$

Also

$$
\int_{0}^{1}\left\|T_{n} f(t, \cdot)\right\|_{p}^{p} d t \geq \int_{|x| \leq c n^{-1 / 3}}\left(\sum_{j}\left|T_{n} f_{j}(x)\right|^{2}\right)^{p / 2} d x \geq c^{2+p} n^{-\frac{2}{3}-\frac{p}{6}},
$$

where the third inequality uses (5). With (6) this yields (1).

A computation shows that $\widehat{T}_{n}(\xi)=e^{i n \arg (\xi)} J_{n}(|\xi|)$ (whence the name of this note). Thus (3) follows from the estimate, uniform in $n$,

$$
\left|J_{n}(r)\right| \leq C r^{-\frac{1}{3}} \quad \text { if } \quad r \geq 1
$$

(see p.357 in $[\mathrm{S}]$ ) combined with the observation

$$
\left|J_{n}(r)\right| \leq \frac{C}{n} \quad \text { if } \quad 0 \leq r \leq \frac{3 n}{4} .
$$


To begin the proof of (4), let $\rho$ be a smooth cutoff function which is equal to 1 on the annulus $\left\{\frac{3}{4} \leq|\xi| \leq \frac{5}{4}\right\}$ and is supported in the annulus $\left\{\frac{1}{2} \leq|\xi| \leq \frac{3}{2}\right\}$. Let $S_{n}$ be the operator defined by $\widehat{S}_{n}(\xi)=\widehat{T}_{n}(\xi) \rho\left(\left|n^{-1} \xi\right|\right)$. The easy estimate

$$
\left|J_{n}(r)\right| \leq C n^{-\frac{1}{2}} \quad \text { if } \quad r \geq \frac{5 n}{4}
$$

combines with (7) to show that the $L^{2}\left(\mathbb{R}^{2}\right)$ operator norm $\left\|T_{n}-S_{n}\right\|_{2,2}$ is $O\left(n^{-\frac{1}{2}}\right)$. Interpolating this with $\left\|T_{n}-S_{n}\right\|_{\infty, \infty}=O(1)$ yields $\left\|T_{n}-S_{n}\right\|_{4,4}=O\left(n^{-\frac{1}{4}}\right)$. Thus (4) will follow from

$$
\left\|S_{n}\right\|_{4,4} \leq C n^{-\frac{1}{4}}(\log (n))^{a},
$$

which is our principal result. The Fourier transform $\widehat{S}_{n}(\xi)$ is supported in the annulus $A_{n}=\left\{\frac{n}{2} \leq|\xi| \leq \frac{3 n}{2}\right\}$. Having fixed $n$, we will decompose $S_{n}$ by decomposing $A_{n}$ into a union of annuli $A_{n}^{j}$ as follows:

$$
\begin{aligned}
\text { for } j \geq 1, \text { set } A_{n}^{j} & =\left\{n+2^{j} n^{\frac{1}{3}} \leq|\xi| \leq n+2^{j+1} n^{\frac{1}{3}}\right\} \\
\operatorname{set} A_{n}^{0} & =\left\{n-2 n^{\frac{1}{3}} \leq|\xi| \leq n+2 n^{\frac{1}{3}}\right\} \\
\text { for } j \leq-1, \text { set } A_{n}^{j} & =\left\{n-2^{|j|+1} n^{\frac{1}{3}} \leq|\xi| \leq n-2^{|j|} n^{\frac{1}{3}}\right\} .
\end{aligned}
$$

Introducing a suitable partition of unity on the Fourier transform side leads to the decomposition

$$
S_{n}=\sum_{j} S_{n}^{j}
$$

For fixed $n$, the number of terms $S_{n}^{j}$ is $O(\log (n))$. Thus (8) will follow from

$$
\left\|S_{n}^{j}\right\|_{4,4} \leq C n^{-\frac{1}{4}}(\log (n))^{b}
$$

for all $j$ and $n$ and some $b>0$. At this point we make a further decomposition of $A_{n}^{j}$ into sectors $A_{n}^{j l}$ of opening angle $\delta \doteq 2^{|j| / 2} n^{-\frac{1}{3}}$. This leads to a decomposition

$$
S_{n}^{j}=\sum_{l=1}^{\delta^{-1}} S_{n}^{j l} .
$$

The function $\widehat{S}_{n}^{j l}$ is supported in a set $R^{j l}$ obtained from the intersection of the annulus $n+\frac{1}{2} n \delta^{2} \leq|\xi| \leq n+3 n \delta^{2}$ with a sector of angle $\delta$; thus, $R^{j l}$ is essentially a rectangle of dimensions $n \delta$ by $n \delta^{2}$, with major dimension $n \delta$ normal to the vector through the center of $R^{j l}$.

\section{Lemma.}

$$
\left\|S_{n}^{j l}\right\|_{4,4} \leq C n^{-\frac{1}{4}} \delta^{\frac{1}{4}} .
$$

Proof. We will obtain the lemma by interpolating the following estimates:

$$
\begin{aligned}
\left\|S_{n}^{j l}\right\|_{2,2} & \leq C(n \delta)^{-\frac{1}{2}}, \\
\left\|S_{n}^{j l}\right\|_{\infty, \infty} & \leq C \delta .
\end{aligned}
$$

The first estimate in (10) is a bound on $J_{n}(r)$ over the annulus $A_{n}^{j}$. The desired estimates are well known, but we provide the simple argument here for completeness. 
For $j=0$, the desired bounds follow from the uniform bound $\left|J_{n}(r)\right| \leq C n^{-\frac{1}{3}}$. For $j \neq 0$, it suffices to show that

$$
\left|\int_{0}^{\pi} e^{i n t-i n\left(1 \pm \delta^{2}\right) \sin t} d t\right| \leq C(n \delta)^{-\frac{1}{2}}
$$

where $C$ is uniform over $n \in \mathbb{Z}$ and $\delta^{2} \leq 1 / 2$.

We let $\phi(t)=t-\left(1 \pm \delta^{2}\right) \sin t$. On the interval $0 \leq t \leq \delta$, we have $\left|\phi^{\prime}(t)\right| \geq c \delta^{2}$, and $\phi^{\prime}(t)$ is monotonic, so Proposition 2 of [S], page 332 , implies that

$$
\left|\int_{0}^{\delta} e^{i n t-i n\left(1 \pm \delta^{2}\right) \sin t} d t\right| \leq C\left(n \delta^{2}\right)^{-1} \leq C(n \delta)^{-\frac{1}{2}}
$$

On the interval $\delta \leq t \leq \pi-\delta$, it follows that $\left|\phi^{\prime \prime}(t)\right| \geq c \delta$, and the same proposition implies that

$$
\left|\int_{\delta}^{\pi-\delta} e^{i n t-i n\left(1 \pm \delta^{2}\right) \sin t} d t\right| \leq C(n \delta)^{-\frac{1}{2}}
$$

On the interval $\pi-\delta \leq t \leq \pi,\left|\phi^{\prime}(t)\right| \geq 1$, and the integral is bounded by $n^{-1}$.

For the second estimate of (10), it suffices to consider the term $S_{n}^{j 0}$, associated to the rectangle $R_{n}^{j 0}$ with center on the positive $\xi_{2}$ axis. The partition of unity element associated to this rectangle is of the form $\widehat{\psi}\left((n \delta)^{-1} \xi_{1},\left(n \delta^{2}\right)^{-1}\left(\xi_{2}-n\right)\right)$, where $\psi$ is a Schwartz function, whose seminorms are bounded by constants independent of $n, j, l$. Thus, the convolution kernel associated to $S_{n}^{j 0}$ is of the form

$$
K_{n}^{j 0}(x)=n^{2} \delta^{3} \int_{-\pi}^{\pi} e^{i n\left(x_{2}-\sin t\right)+i n t} \psi\left(n \delta\left(x_{1}-\cos t\right), n \delta^{2}\left(x_{2}-\sin t\right)\right) d t .
$$

We need to show that

$$
\int\left|K_{n}^{j 0}(x)\right| d x \leq C \delta .
$$

The contribution from the integral over $|t| \leq \delta$ trivially satisfies (11), so it suffices to consider the following term:

$$
\widetilde{K}(x)=n^{2} \delta^{3} \int e^{i n(t-\sin t)} \chi\left(\delta^{-1} t\right) \psi\left(n \delta\left(x_{1}-\cos t\right), n \delta^{2}\left(x_{2}-\sin t\right)\right) d t,
$$

where $\chi(s)=1$ for $|s| \geq 2$, and $\chi(s)=0$ for $|s| \leq 1$. Integration by parts yields

$$
\widetilde{K}(x)=i n \delta^{3} \int e^{i n(t-\sin t)} \frac{\partial}{\partial t}\left[\frac{\chi\left(\delta^{-1} t\right)}{1-\cos t} \psi\left(n \delta\left(x_{1}-\cos t\right), n \delta^{2}\left(x_{2}-\sin t\right)\right)\right] d t .
$$

The term where the derivative falls on the term in front of $\psi$ satisfies (11), since

$$
\int\left|\frac{\partial}{\partial t}\left(\frac{\chi\left(\delta^{-1} t\right)}{1-\cos t}\right)\right| d t \leq C \delta^{-2} \leq C n \delta .
$$

The term where the derivative falls on the $x_{2}$ place of $\psi$ also satisfies (11), since

$$
\int\left|\frac{\chi\left(\delta^{-1} t\right) \cos t}{1-\cos t}\right| d t \leq C \delta^{-1}
$$

The term where the derivative falls on the $x_{1}$ place of $\psi$ would appear to lead to bounds comparable to $\delta \log \left(\delta^{-1}\right)$; however, one further integration by parts shows that this term too satisfies (11). 
We now prove (9) by noting that the angle $\delta$ was chosen so that the sets $R^{j l}+R^{j l^{\prime}}$ have bounded overlap for $R^{j l}$ and $R^{j l^{\prime}}$ in the same quadrant, i.e., so that the orthogonality argument of $[\mathrm{F}]$ applies. This argument yields

$$
\left\|\sum_{l} S_{n}^{j l} f\right\|_{4} \leq C\left\|\left(\sum_{l}\left|S_{n}^{j l} f\right|^{2}\right)^{\frac{1}{2}}\right\|_{4} \text {. }
$$

The number of indices $l$ is $O\left(\delta^{-1}\right)$, so

$$
\sum_{l}\left|S_{n}^{j l} f(x)\right|^{2} \leq C \delta^{-\frac{1}{2}}\left(\sum_{l}\left|S_{n}^{j l} f(x)\right|^{4}\right)^{\frac{1}{2}} .
$$

With $\widehat{f}_{j l}$ representing the localisation of $\widehat{f}$ to an appropriate sector, we thus have

$$
\begin{aligned}
\left\|\sum_{l} S_{n}^{j l} f\right\|_{4} & \leq C \delta^{-\frac{1}{4}}\left\|\left(\sum_{l}\left|S_{n}^{j l} f\right|^{4}\right)^{\frac{1}{4}}\right\|_{4} \\
& \leq C n^{-\frac{1}{4}}\left\|\left(\sum_{l}\left|f_{j l}\right|^{4}\right)^{\frac{1}{4}}\right\|_{4} \\
& \leq C n^{-\frac{1}{4}}\left\|\left(\sum_{l}\left|f_{j l}\right|^{2}\right)^{\frac{1}{2}}\right\|_{4} .
\end{aligned}
$$

A result of Córdoba $[\mathrm{C}]$ gives

$$
\left\|\left(\sum_{l}\left|f_{j l}\right|^{2}\right)^{\frac{1}{2}}\right\|_{4} \leq C(\log (n))^{b}\|f\|_{4}
$$

for some positive $b$, which completes the proof of (9).

\section{REFERENCES}

[C] A. Córdoba, Geometric Fourier Analysis, vol. 32, Ann. Inst. Fourier, 1982, pp. 215-226. MR 84i: 42029

[F] C. Fefferman, A note on spherical summation multipliers, Israel J. Math 15 (1973), 44-52. MR 47:9169

[O] D. Oberlin, Convolution estimates for some measures on curves, Proc. Amer. Math. Soc 99 (1987), 56-60.

[SS] H. F. Smith and C. D. Sogge, $L^{p}$ regularity for the wave equation with strictly convex obstacles, Duke Math. J. 73 (1994), 97-153. MR 95c:35048

[S] E. M. Stein, Harmonic Analysis: Real Variable Methods, Orthogonality, and Oscillatory Integrals, Princeton University Press, Princeton, 1993.

[SW] E. M. Stein and S. Wainger, Problems in harmonic analysis related to curvature, Bull. Amer. Math. Soc. 84 (1978), 1239-1295. MR 80k:42033

Department of Mathematics, Florida State University, Tallahassee, Florida 32306

E-mail address: oberlin@math.fsu.edu

Department of Mathematics, University of Washington, Seattle, Washington 98195

E-mail address: hart@math.washington.edu 